The ASTROPHYSiCal Journal Supplement Series, 90:701-705, 1994 February

(c) 1994. The American Astronomical Society. All rights reserved. Printed in U.S.A.

\title{
POSITRON ANNIHILATION RADIATION FROM THE 1991 NOVEMBER 15 FLARE
}

\author{
K. Kawabata \\ Department of Physics and Astrophysics, Nagoya University, Chikusa-ku, Nagoya 464-01, Japan \\ M. Yoshimori, K. Suga, K. Morimoto, T. Hiraoka, and J. Sato \\ Department of Physics, Rikkyo University, 3 Nishi-Ikebukuro, Toshima-ku, Tokyo 171, Japan
}

AND

K. OHKI

National Astronomical Observatory, 2-21-1 Osawa, Mitaka, Tokyo 181, Japan

Received 1993 March 1: received 1993 July 26

\begin{abstract}
The gamma-ray spectrometer on Yohkoh has detected the positron annihilation line at $511 \mathrm{keV}$ produced during the 1991 November 15 flare (X1.0/3B). The $511 \mathrm{keV}$ line fluence, integrated over the time interval of 22:37:50-22:38:14 UT, is $(6.7 \pm 2.2)$ photons $\mathrm{cm}^{-2}$. The time profile of $511 \mathrm{keV}$ line exhibited long decay time compared with the electron bremsstrahlung and prompt gamma-ray line components. From the analysis of time profile of the $511 \mathrm{keV}$ line, we come to the following conclusions: (1) the main source of positrons is deexcitation of ${ }^{16} \mathrm{O}^{* 6.052}$ by $e^{+}-e^{-}$pair emission. (2) $\beta^{+}$-emitting nuclei of ${ }^{31} \mathrm{~S},{ }^{29} \mathrm{P},{ }^{27} \mathrm{Si},{ }^{26 \mathrm{~m}} \mathrm{Al},{ }^{25} \mathrm{Al},{ }^{23} \mathrm{Mg},{ }^{19} \mathrm{Ne}$, and ${ }^{21} \mathrm{Na}$ are also important sources of positrons in the decay phase. (3) The density of the positron annihilation region in the photosphere is $10^{16} \mathrm{~cm}^{-3}$. (4) Most likely interpretation of the time profile is that at least $50 \%$ of positrons annihilate in coronal flare loops with a density of $10^{12}-10^{13} \mathrm{~cm}^{-3}$ and with a temperature of $10^{6}-3 \times 10^{6} \mathrm{~K}$.
\end{abstract}

Subject headings: nuclear reactions, nucleosynthesis, abundances - Sun: flares - Sun: X-rays, gamma-rays

\section{INTRODUCTION}

Positrons in solar flares are primarily produced by radioactive $\beta^{+}$-emitting nuclei, deexcitation by $e^{+}-e^{-}$pair emission, and decay of $\pi^{+}$mesons produced by nuclear interactions between accelerated ions and ambient solar material (Ramaty, Kozlovsky, \& Lingenfelter 1975). The positron production from the decay of various radioactive nuclei, from the deexcitation due to the $e^{+}-e^{--}$pair emission, and from the decay of $\pi^{+}$meson have been studied in detail by Kozlovsky, Lingenfelter, \& Ramaty (1987), hereafter KLR). The ambient density and temperature determine the relative contribution of free annihilation and decay of positronium, as well as the width of the annihilation line (Crannell et al. 1976, hereafter CJRW; Bussard, Ramaty, \& Drachman 1979, hereafter BRD).

Positron annihilation line at $511 \mathrm{keV}$ from solar flares was first detected with a gamma-ray spectrometer on $O S O 7$ during the solar flare on 1972 August 4 (Chupp et al. 1973). The excess of $511 \mathrm{keV}$ line was $\sim 3.1 \sigma$ above the continuum and the fluence was $\sim 35$ photons $\mathrm{cm}^{-2}$. In the last solar maximum, the strong $511 \mathrm{keV}$ line was emitted during two intense solar flares on 1980 June 21 and 1982 June 3 and more detailed observations have been made with the powerful gammaray spectrometer on the Solar Maximum Mission (Share et al. 1983). The time profile of the $511 \mathrm{keV}$ line during the 1980 June 21 flare is consistent with a calculation (Murphy \& Ramaty 1984).

In this paper, we present an observation of positron annihilation radiation from the 1991 November 15 flare observed with the Yohkoh gamma-ray spectrometer. Positron emitters responsible for emission of annihilation line, propagation of positrons in the solar atmosphere, effects of positronium for- mation, and the probable location of annihilation region are discussed on the basis of the observations and calculations.

\section{OBSERVATION}

The Yohkoh hard X-ray and gamma-ray spectrometers detected an X-class flare at 22:37 UT on 1991 November 15. The flare location was S12W13, and the GOES class and $\mathrm{H} \alpha$ importance were $1.0 \mathrm{X}$ and $3 \mathrm{~B}$, respectively. The primary-data output of the Yohkoh gamma-ray spectrometer is a 128 channel pulse height spectrum from 0.4 to $100 \mathrm{MeV}$ every $4 \mathrm{~s}$. The instrument description has been given in detail by Yoshimori et al. (1991).

The time profiles of counting rate in the 502-532, 418-473 $\mathrm{keV}$, and 4-7 MeV bands are shown in Figure 1 in order to compare the time profile of the $511 \mathrm{keV}$ line with those of the electron bremsstrahlung and of prompt nuclear lines. The time profiles are plotted with a $4 \mathrm{~s}$ time resolution. The $418-473$ $\mathrm{keV}$ band emission is dominated by the electron bremsstrahlung, but is partly due to $\mathrm{Li}$ and $\mathrm{Be}$ lines around 22:37:48 UT (Yoshimori 1993). The 4-7 MeV band emission is dominated by prompt nuclear deexcitation lines of ${ }^{12} \mathrm{C}$ and ${ }^{16} \mathrm{O}$ at 4.44 and $6.12 \mathrm{MeV}$. The $502-532 \mathrm{keV}$ emission is composed of the electron bremsstrahlung and the $511 \mathrm{keV}$ line. Gamma-ray emission in the 4-7 MeV band started to enhance at 22:37:32 UT, attained the maximum at 22:37:52 UT, and lasted until 22:38:00 UT. The emission in the 418-473 keV band shows similar temporal variations to that in the 4-7 MeV band. Although the emission in 502-532 keV band also shows similar temporal variations to those of the 4-7 MeV and 418-473 keV bands before the peak time (22:37:50 UT), it shows gradual characteristics in the decay phase compared with the other two energy bands. 


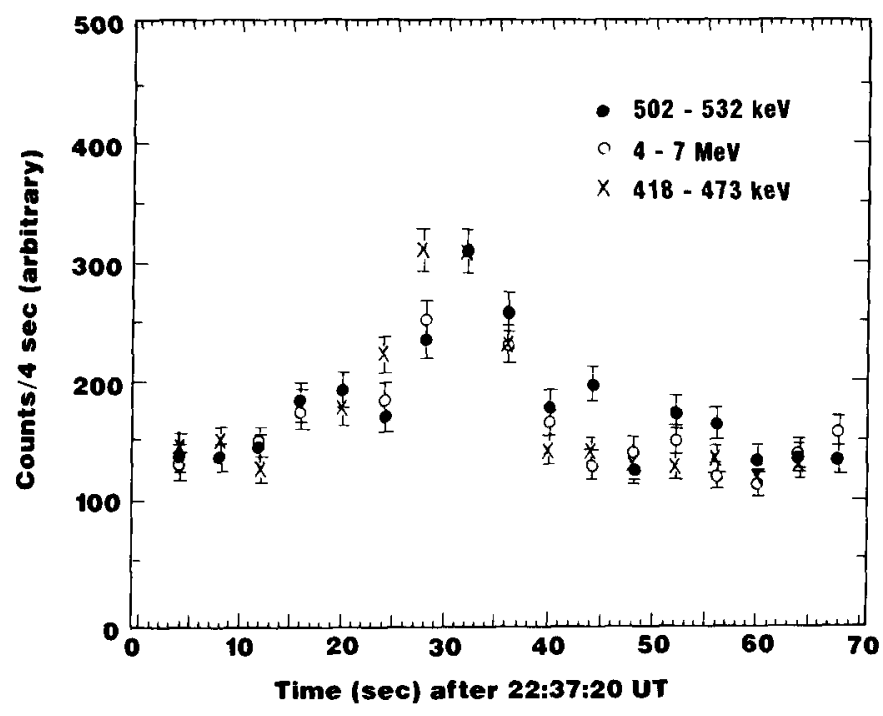

FIG. 1.-The time profiles of counting rates for $502-532 \mathrm{keV}, 4-7$ $\mathrm{MeV}$, and 418-473 keV band emission.

Background-subtracted count spectra in the $400-800 \mathrm{keV}$ band, integrated for 12,24 , and $36 \mathrm{~s}$ from 22:37:50 UT, are illustrated in Figure 2. The $511 \mathrm{keV}$ line is apparent above the continuum in these spectra. The background count spectrum measured in the time interval of 22:34:00 to $22: 36: 00$ UT is used to derive the background-subtracted count spectra. The $511 \mathrm{keV}$ line fluence, integrated over the time interval of 22:37:50-22:38:14 UT, is $(6.7 \pm 2.2)$ photons $\mathrm{cm}^{-2}$.

Comparison of two line widths obtained from the solar flare and background spectra enable us to establish an upper limit to the intrinsic line width of the solar positron annihilation line. The upper limit is $20 \mathrm{keV}$ in FWHM. This allows us to set the upper limit to the temperature in the annihilation region as $3 \times$ $10^{6} \mathrm{~K}$.

In the following, the time variation of annihilation line is discussed on the bases of time integrated counts as a function of integration time, because these have higher $\mathrm{S} / \mathrm{N}$ ratio than annihilation line counting rates. The time-integrated counts in $502-532 \mathrm{keV}$ band (filled circle with rms error bars) and of bremsstrahlung component (open circle) obtained from interpolations of count spectra are plotted versus the integration time in Figure 3.

\section{DISCUSSION AND CONCLUSIONS}

Because the gamma-ray spectrum in the 4-7 MeV band is dominated by prompt nuclear lines, the time profile of counting rates in 4-7 MeV band represents the time variation of the number of accelerated nuclei impinging on the solar atmosphere. We have calculated the time-integrated counts as a function of integration time, assuming that the precipitation rate is proportional to the counting rate of the prompt gammarays $(4-7 \mathrm{MeV})$. The production yields of each positron emitter depends on the energy spectrum of impinging energetic nuclei and interaction models. Concerning the angular distribution of the interacting particles, Yoshimori (1993) has shown that a spectral feature composed of the Be and Li lines appears to support a strong pitch-angle scattering in the corona
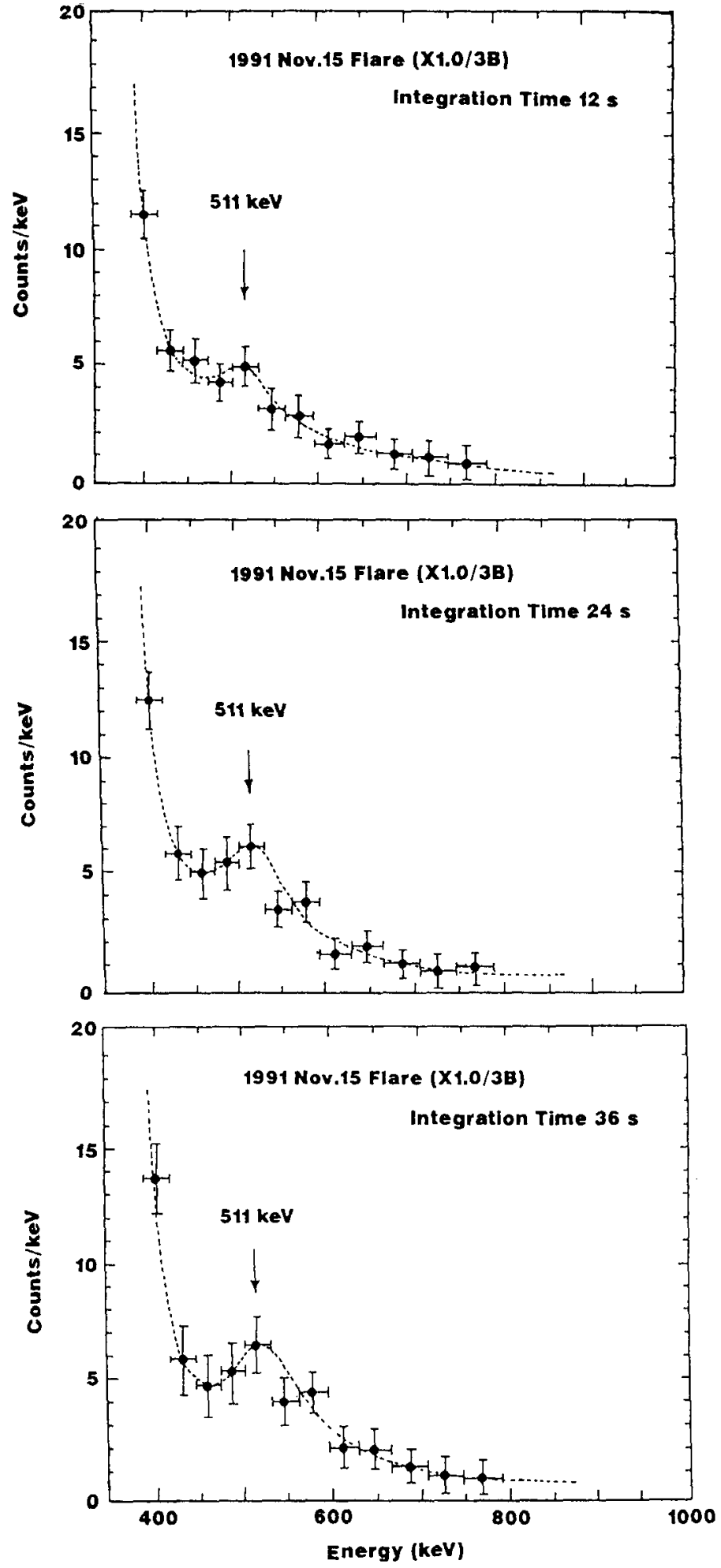

FIG. 2.- $\gamma$-ray spectrum between 400 and $800 \mathrm{keV}$ integrated from $22: 37: 50$ through $22: 38: 02,22: 38: 14$, and 22:38:26 UT, respectively. 

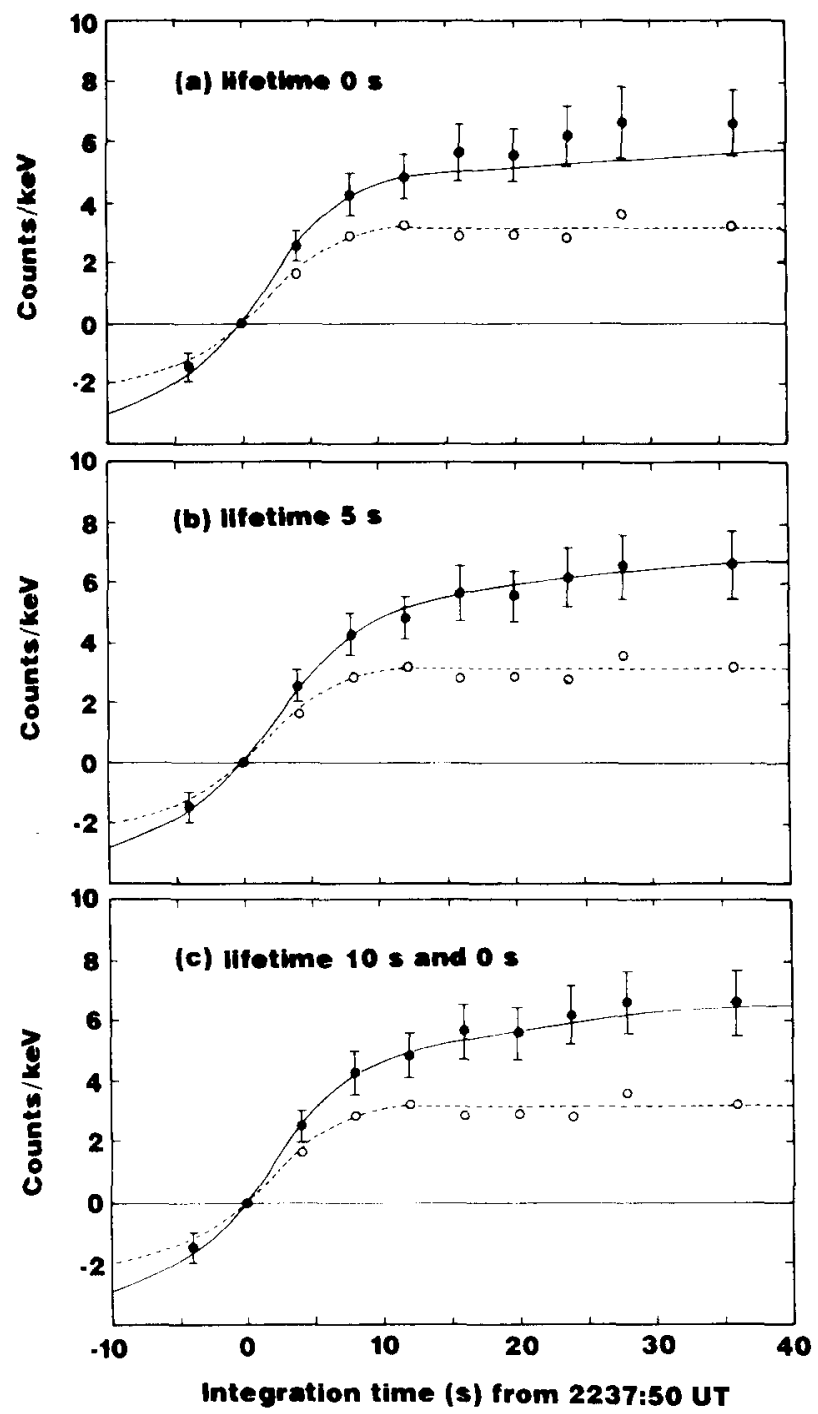

FIG. 3.- Integrated counts of $511-532 \mathrm{keV}$ band (filled circle) and of bremsstrahlung (open circle) from 22:37:50 UT. Solid line: theoretical counts of summation of annihilation line and bremsstrahlung. Dotted line: theoretical counts of bremsstrahlung.

(a downward-peaked distribution of interacting particles) (Murphy et al. 1990). The observation supports the thick-target model as an interaction model. The ratio of fluence of 4.44 and $2.22 \mathrm{MeV}$ line gives the acceleration parameter $\alpha T=$ $0.009 \pm 0.002$ ( $\alpha$ is the acceleration rate, and $T$ is the escape time from the acceleration region ) in the modified Bessel function representation of stochastic acceleration spectrum ( $\mathrm{Ra}-$ maty 1979). In the following calculation, we have adopted production yields in the thick-target model for $\alpha T=0.01$ in KLR. Positron emitters with mean life less than $2 \mathrm{hr}$ in KLR are taken into account in the calculation except those with very small yields. Positron emitters included in the calculation are ${ }^{16} \mathrm{O}^{* 6.052}$ ("*6.052" means the excited level of ${ }^{16} \mathrm{O}$ nucleus with an excitation energy of $6.052 \mathrm{MeV}),{ }^{12} \mathrm{~N},{ }^{548} \mathrm{Co},{ }^{28} \mathrm{P},{ }^{32} \mathrm{Cl}$, ${ }^{20} \mathrm{Na},{ }^{39} \mathrm{Ca},{ }^{37} \mathrm{~K},{ }^{34 \mathrm{~g}} \mathrm{Cl},{ }^{35} \mathrm{Ar},{ }^{24} \mathrm{Al},{ }^{33} \mathrm{Cl},{ }^{31} \mathrm{~S},{ }^{29} \mathrm{P},{ }^{27} \mathrm{Si},{ }^{26 \mathrm{~m}} \mathrm{Al},{ }^{25} \mathrm{Al}$, ${ }^{23} \mathrm{Mg},{ }^{19} \mathrm{Ne},{ }^{10} \mathrm{C},{ }^{21} \mathrm{Na},{ }^{17} \mathrm{~F},{ }^{14} \mathrm{O},{ }^{54 \mathrm{~m}} \mathrm{Co},{ }^{15} \mathrm{O},{ }^{30} \mathrm{P},{ }^{53} \mathrm{Fe},{ }^{13} \mathrm{~N}$, and ${ }^{11} \mathrm{C}$.

Because the duration of main phase of annihilation line emission was only $10 \mathrm{~s}$ or so, principal positron emitters responsible for emission of the annihilation line must have mean lives less than $10 \mathrm{~s}$. Among the positron emitters with mean lives less than $10 \mathrm{~s}$ in the table of production yields in KLR, only ${ }^{16} \mathrm{O}^{* 6.052}$ has a large production yield. Therefore, positrons are produced mainly by the $e^{+}-e^{-}$pair emission from ${ }^{16} \mathrm{O} * 6.052$. Other positron emitters produce $25 \%-30 \%$ of positrons in the precipitation phase. Figure 3 illustrates also the calculated counts of bremsstrahlung component in 502-532 $\mathrm{keV}$ band (dotted lines) and the summation of calculated counts of bremsstrahlung and annihilation line (the solid line) in three cases. These calculated values are normalized to observed value at the integration time of $8 \mathrm{~s}$. Here we assume bremsstrahlung is proportional to $4-7 \mathrm{MeV}$ band counting rates. We can find a good agreement of calculated integrated counts of bremsstrahlung with observed values in Figure 3.

In Figure $3 a$, the calculation is carried out by assuming positrons annihilate in the photosphere. In this case, the mean life of positrons is very short in comparison with the time resolution of observations. $\beta^{+}$-emitting nuclei of ${ }^{31} \mathrm{~S},{ }^{29} \mathrm{P},{ }^{27} \mathrm{Si},{ }^{26 \mathrm{~m}} \mathrm{Al}$, ${ }^{25} \mathrm{Al},{ }^{23} \mathrm{Mg},{ }^{19} \mathrm{Ne}$, and ${ }^{21} \mathrm{Na}$ with life time less than $60 \mathrm{~s}$ are important positron emitters in the decay phase between 22:38:02 and 22:38:26 UT. These radio nuclei are mainly produced by proton and $\alpha$ interactions with ${ }^{20} \mathrm{Ne},{ }^{24} \mathrm{Mg},{ }^{28} \mathrm{Si}$, and ${ }^{32} \mathrm{~S}$. The $502-532 \mathrm{keV}$ counts obtained by integration more than $16 \mathrm{~s}$ from 22:37:50 UT deviates systematically from the calculation in Figure $3 a$, indicating that the temporal variation of the $511 \mathrm{keV}$ emission cannot be interpreted by the decay time of $\beta^{+}$emitters alone. An overabundance of $\mathrm{Ne}, \mathrm{Mg}, \mathrm{Si}$, and $S$ will be a possible interpretation of the discrepancy of the temporal variation of the $511 \mathrm{keV}$ line. Our calculation, however, shows that an overabundance by a factor of 5-10 must be assumed in comparison with the Cameron's abundances (1982) for the interpretation. Therefore, the interpretation is unlikely. A likely cause of the discrepancy is that the assumption of the annihilation in the photosphere alone is incorrect. In this case, positrons may have significant mean life. The calculated line in Figure $3 b$ is obtained by assuming the mean life of $5 \mathrm{~s}$ for positrons. The calculation shows a good agreement with the observations.

For $\alpha T=0.01$, production of ${ }^{16} \mathrm{O} * 6.052$ is mostly due to proton reaction which dominates $\alpha$ particle reactions by an order of magnitude. Energetic particles interact with ambient nuclei after deceleration. Figure 4 illustrates the production rate $\left(\mathrm{cm}^{-1}\right)$ of ${ }^{16} \mathrm{O}^{* 6.052}$ for the proton reaction as a function of the penetration depth $\left(\mathrm{g} \mathrm{cm}^{-2}\right)$ for several injection energies. We have used the cross section for the nuclear reaction in KLR and the formula of ionization loss in the atmospheric atoms (Ginzburg \& Syrovatskii 1964, p. 121). In this calculation, we have also assumed the $\mathrm{He}: \mathrm{H}$ ratio of $1: 10$.

The effective incident energy of protons for the production of ${ }^{16} \mathrm{O} * 6.052$ is $\sim 10 \mathrm{MeV}$ for $\alpha T=0.01$. Figure 4 gives the penetration depth of $0.03 \mathrm{~g} \mathrm{~cm}^{-2}$ for the peak of the production rates of ${ }^{16} \mathrm{O}^{* 6.052}$. The number density of atoms in model $\mathrm{C}$ (the average quiet Sun) of Vernazza, Avrett, \& Loser (1981, hereafter VAL) model at the penetration depth is $10^{15} \mathrm{~cm}^{-3}$. Production layers of the other positron emitters important for the present case are almost the same as ${ }^{16} \mathrm{O} * 6.052$ because threshold energies for interactions do not differ significantly. Recent submillimeter observations during the $1991 \mathrm{July} 11$ total solar 


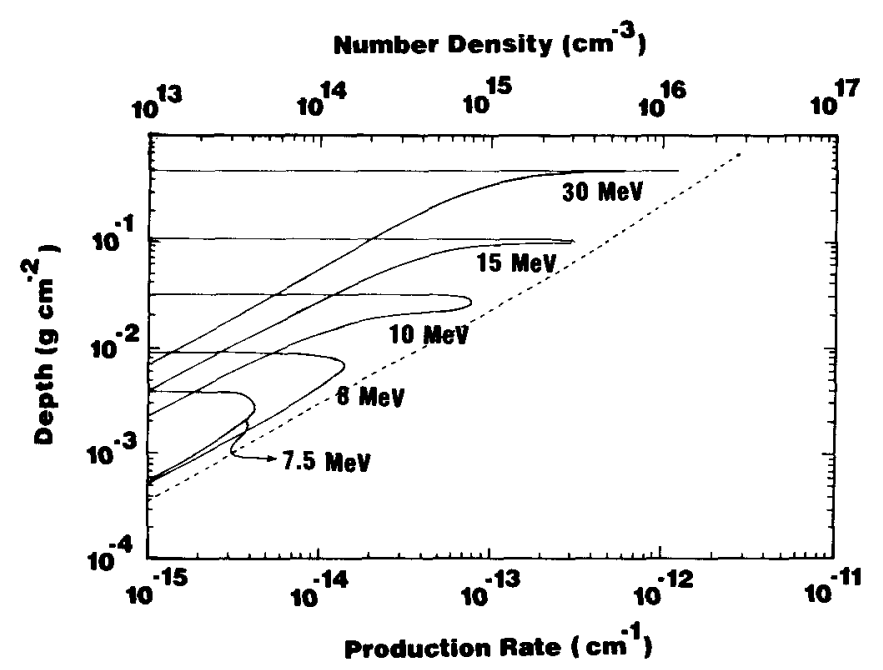

FIG. 4.-Production rate of ${ }^{16} \mathrm{O}^{* 6.052}$ for proton reaction as a function of the depth in $\mathrm{g} \mathrm{cm}^{-2}$ for selected incident energies indicated in the figure in a unit of MeV. The number density of the atoms (VAL model) at the corresponding depth for the vertical incidence is also shown by a dotted line.

eclipse (Ewell et al. 1993) have indicated that the upper chromosphere is not in hydrostatic equilibrium and has higher density than VAL model. In the model by Ewell et al., the number density at a given penetration depth is low compared with that of the VAL model by a factor of $\sim 3$.

If we assume a uniform magnetic field in the photosphere, half of positrons produced penetrates downward because of symmetric emission from the positron emitters and annihilates in the photosphere after the deceleration. The remainder of positrons moves upward and escapes to the corona without significant energy loss. The ratio of the number of positrons moving downward to upward depends on the configuration of the magnetic field in the production layer of positron emitters and below. Positrons escaping to the corona can again impinge the photosphere by mirror effect or at the other footpoint. Another plausible situation is that these positrons are trapped and annihilate in the magnetic loop spatially in the corona. The ratio of the number of positrons annihilating in the photosphere to that in the magnetic loop depends on the configuration of the magnetic field, the density, wave-particle interactions, and so on, in the loop.

Positrons due to $e^{+}-e^{-}$pair emission have an energy of 2.5 $\mathrm{MeV}$ and those due to the $\beta^{+}$decay have energies a little less than $1 \mathrm{MeV}$. The average depths of the annihilation region in the photosphere are 0.6 and $0.2 \mathrm{~g} \mathrm{~cm}^{-2}$, for positrons with an energy of 2.5 and $1 \mathrm{MeV}$, respectively. The density of annihilation region in the photosphere becomes $2 \times 10^{16} \mathrm{~cm}^{-3}$ and $1 \times$ $10^{16} \mathrm{~cm}^{-3}$, respectively, according to the VAL model in the case of uniform magnetic filed in the photosphere. When positrons are reflected by mirror effect, the density of the annihilation region becomes lower but must be higher than that of the production layer, $10^{15} \mathrm{~cm}^{-3}$. At these densities, the following points have been indicated by CJRW and BRD. (1) Positrons form positronium before free annihilation due to the charge exchange. (2) Positronium in ' $S_{0}$ state annihilates into $2 \gamma$ emission. (3) Positronium in ${ }^{3} S_{1}$ state are ionized or flipped to ${ }^{1} S_{0}$ state through collisions with free electrons before $3 \gamma$ emis- sion. The deceleration and annihilation time are very short compared with the time resolution of the gamma-ray spectrometer on Yohkoh for the annihilation in the photosphere.

The optical depths to the Compton scattering in the annihilation line become 0.2 and 0.07 at the depth of 0.6 and $0.2 \mathrm{~g}$ $\mathrm{cm}^{-2}$, respectively, for the vertical emission toward Earth. The annihilation line due to the decay of ${ }^{16} \mathrm{O}^{* 6.052}$ suffers strong attenuation for flares with a central distance more than $78^{\circ}$ or $0.98 R_{\odot}$. For positrons due to $\beta^{+}$decay, the attenuation of annihilation line becomes significant at the central distance of $86^{\circ}$, or $0.998 R_{\odot}$. The annihilation in the photosphere alone has difficulty against interpretations of limb event such as the 1980 June 21 event because of strong attenuation of annihilation line due to the Compton scattering.

The remainder of positrons moves upward and escapes to the corona without significant energy loss. These positrons can be trapped and annihilate in the corona. Hudson et al. (1992) have observed a white-light flare of a looplike structure with Soft X-ray Telescope on Yohkoh during the present flare. The peak brightness in white light occurred at 22:37:42 UT. They have suggested that sufficient material evolved into the corona to provide enough opacity at an altitude of $8,000 \mathrm{~km}$. The white-light image suggests that the length of a white-light loop is $-24,000 \mathrm{~km}$. Then the traveling time of relativistic positrons to the opposite footpoint is the order of $0.1 \mathrm{~s}$. The traveling time is much less than the time resolution of the Yohkoh gamma-ray spectrometer. Feldman (1992) has suggested that the electron density of flare coronal loops may be as high as $10^{13} \mathrm{~cm}^{-3}$. Hiei, Nakagomi, \& Takuma (1992, hereafter HNT) have obtained the electron density of $10^{12}-10^{13} \mathrm{~cm}^{-3}$ and the temperature of $10^{4}-10^{7} \mathrm{~K}$ for a loop prominence system observed in white light associated with a flare on 1989 August 16.

The temperature of the dense coronal loops responsible for the annihilation must be less than $3 \times 10^{6} \mathrm{~K}$ from the observed line width of the annihilation line and must be higher than $10^{6}$ $\mathrm{K}$ for free annihilation according to the calculation by BRD. As is shown later, the fluence of the annihilation line is in agreement with a calculation from prompt lines, only if positrons annihilate directly with free electrons. The deceleration time of positrons having an energy of $2.5 \mathrm{MeV}$ is approximately given by $7 \times 10^{12} / n_{e}$ s in a fully ionized plasma with an electron density $n_{e}$ between $10^{11}$ and $10^{14} \mathrm{~cm}^{-3}$ according to the formula given by Ginzburg \& Syrovatskii (1964). The deceleration time becomes shorter as the energy of positron decreases. The deceleration time is an order of magnitude shorter than the lifetime of positron annihilation $5 \times 10^{13} / n_{e} \mathrm{~s}$ obtained by CJRW and BRD, taking into account Coulomb correction. If we put the lifetime of positrons as $5 \mathrm{~s}$, the electron density of the annihilation region becomes $10^{13} \mathrm{~cm}^{-3}$. In Figure $3 c$, we assume half of positrons annihilates in the photosphere with a lifetime of $0 \mathrm{~s}$, and the remainder annihilates in the coronal region with a lifetime of $10 \mathrm{~s}$. Agreement with the observation can be obtained, when we assume the life time of positrons in the corona between 10 and $50 \mathrm{~s}$. We come to the conclusions that the agreement with the observation can be obtained, only if more than $50 \%$ of positrons have a lifetime of $5-50 \mathrm{~s}$.

The number of protons impinging on the photosphere can be obtained from fluencies of the ${ }^{12} \mathrm{C}$ and ${ }^{16} \mathrm{O}$ lines at 4.44 and $6.13 \mathrm{MeV}$. The method gives $1.1 \times 10^{32}$ as a total number of 
$>30 \mathrm{MeV}$ protons impinging on the photosphere. The number of protons gives the fluence of 5 photons $\mathrm{cm}^{-2}$ integrated from $22: 37: 50$ through 22:38:14 UT, when more than half of positrons decays in the corona by free annihilation with a mean life of 5-10 s. The calculated fluence is in agreement with observed value of $(6.7 \pm 2.2)$ photons $\mathrm{cm}^{-2}$ within observational errors. If a large fraction of positrons in the corona forms positronium, the calculated fluence becomes less than 3 photons $\mathrm{cm}^{-2}$ and is not in agreement with the observation. The total energy of these $>30 \mathrm{MeV}$ protons is $7 \times 10^{27} \mathrm{ergs}$. When we extrapolate the energy spectrum to less than $1 \mathrm{MeV}$, the total energy of energetic nuclei including $\alpha$ particles becomes $1.4 \times 10^{30}$ ergs.

From these considerations, we come to the following conclusions. The most likely annihilation region is the photosphere of density of $10^{16} \mathrm{~cm}^{-3}$ and dense coronal loops such as suggested by Feldman (1992) and HNT at the temperature of
$10^{6}-3 \times 10^{6} \mathrm{~K}$. At least $50 \%$ of positrons annihilates in the dense coronal loop. The electron density of coronal annihilation region obtained is the highest value of $10^{13} \mathrm{~cm}^{-3}$, when all the positrons annihilate in the coronal region. When $50 \%$ of positrons annihilates in the coronal region and the remainder annihilates in the photosphere, good agreement with the observations can be obtained for the electron density of $10^{12}-5 \times$ $10^{12} \mathrm{~cm}^{-3}$.

We would like to thank the staff of the Institute of Space and Astronautical Science, the staff of the NASA Deep Space Network, and all of the Yohkoh team members for their support of the spacecraft operations. This work is supported by Grant-inAid for Scientific Research of Ministry of Education, Science, and Culture (No. 03640285 and 04640311 ), and the Rikkyo University Grant for the Promotion of Research.

Bussard, R. W., Ramaty, R., \& Drachman, R. J. 1979, ApJ, 228, 928 (BRD)

Cameron, A. G. 1982, in Essays in Nuclear Astrophysics, ed. C. Barnes, D. D. Clayton, \& D. N. Schramm (Cambridge Univ. Press), 23

Chupp, E. L., Forrest, D. J., Higbie, P. R., Suri, A. N., Tsai, C., \& Dunphy, P. P. 1973, Nature, 241, 333

Crannell, C. J., Joyce, G., Ramaty, R., \& Werntz, C. 1976, ApJ, 210, 582 (CJRW)

Ewell, M. W., Zirin, H., Jensen, J. B., \& Bastian, T. S. 1993, ApJ, 403, 426

Feldman, U. 1992, preprint

Ginzburg, V. L., \& Syrovatskii, S. I. 1964, The Origin of Cosmic Rays (London: Pergamon)

Hiei, E., Nakagomi, Y., \& Takuma, H. 1992, PASJ, 44, 55 (HNT)

Hudson, H. S., Acton, L. W., Hirayama, T., \& Uchida, Y. 1992, PASJ, 44, L77

Kozlovsky, B., Lingenfelter, R. E., \& Ramaty, R. 1987, ApJ, 316, 801

(KLR)

\section{EFERENCES}

Murphy, R. J., \& Ramaty, R. 1984, Adv. Space Res., 4, No. 7, 127

Murphy, R. J., Hua, X.-M., Kozlovsky, B., \& Ramaty, R. 1990, ApJ, 351, 299

Ramaty, R. 1979, in Particle Acceleration Mechanisms in Astrophysics, ed. J. Arons, C. Max, \& C. Mckee (New York: AIP), 135

Ramaty, R., Kozlovsky, B., \& Lingenfelter, R. E. 1975, Space Science Rev., 18, 341

Share, G. H., Chupp, E. L., Forrest, D. J., \& Rieger, E. 1983, in Positron and Electron Pairs in Astrophysics, ed. M. L. Burns, A. K. Harding, \& R. Ramaty (New York: AIP), 15

Vernazza, J. E., Avrett, E. H., \& Loeser, R. 1981, ApJS, 45, 635

Yoshimori, M. 1993, in Proc. 4th Internat. Conf. on Plasma Physics and Controlled Nuclear Fusion (Toki) (ESA SP-351), 211

Yoshimori, M., et al. 1991, Sol. Phys., 136, 69 